\title{
Critical Behavior of Models with Infinitely Many Absorbing States
}

\author{
J.F.F. Mendes \\ Departamento de Física and Centro de Física do Porto, Universidade do Porto, \\ Rua do Campo Alegre 687, 4169-007 Porto, Portugal
}

Received 1 November 1999

\begin{abstract}
In this article we review several models with many absorbing configurations. In all these models attention will be focused on the influence of the initial state on the dynamic evolution. The relation of systems with many absorbing configurations to those displaying avalanches (self-organized criticality) is also investigated. Some new results are presented both for systems with and without parity conservation. Recently derived scaling relations are tested.
\end{abstract}

\section{Introduction}

The study of nonequilibrium models exhibiting phase transitions from an active to an absorbing phase has been a topic of intense research in the last years [111] since Broadbent and Hammersley [12] introduced directed percolation (DP) [13-15]. Later studies have connected this to other fields, and the topic has become familiar in many processes encountered in nature. Among them we refer to models of epidemics [19-21], catalytic kinetics [22], surface growth [23], and selforganized criticality [24]. Most of these models were found to belong to the universality class of DP, supporting the conjecture of Jenssen [25] and Grassberger [26] that all systems exhibiting a continuous transition into a unique absorbing state, without any other symmetry or conservation law, belong to the DP universality class. This conjecture was later extended by Grinstein et al. [27] to systems with multiple components. Exceptions to this universality class were introduced by Grassberger [28,29]; they correspond to models with doubly degenerated absorbing states and modulo 2 parity conservation.

More recently Jensen [30] introduced a model (the pair contact process - PCP) that is the prototype of a model with a number of absorbing configurations growing with the system size, which goes to infinity in the thermodynamic limit. Other models with infinitely many absorbing states (IMAS) were introduced since then, and have proved to have a more complex dynamics than the ones with only a single absorbing state. Apart from this, all them of show - in agreement with the previous conjecture - a DP-like behavior in what concerns the static critical behavior. However, the critical exponents that govern the spreading from an initial seed (the closest configuration to the absorbing one) show non-universal behavior. The critical exponents describing time dependence depend on the initial configuration of the system. Only for the "natural" configuration (the one obtained from the evolution of the system) are DP values recovered. An attempt to justify this behavior on solid grounds was made by Muñoz et al. [31] using a continuous field theory. Other features of IMAS models were discovered recently by Lipowski [32], who observed that a model inspiried by "biological evolution" in $(2+1)$-dimensions has the critical behavior of $(1+1)$-dimensional DP (an effect similar to the dimensional reduction encountered in random systems [33]).

Another topic of intense research in our days is selforganized criticality (SOC). Recently some attempts to connect $\mathrm{SOC}$ with absorbing-state phase transitions were made $[34,35]$. SOC was introduced by Bak, Tang and Weisenfeld (BTW) [36], who proposed a model for the self-organization of a sandpile. The pile obtained by adding single grains self-organizes to the critical angle, where infinitely large avalanches (by avalanche we understand a successive sequence of toppling events) can occur. This process will reduce again the slope of the pile, which will evolve through small avalanches until it again reaches again the critical point. A very good pedagogical review on this topic is given by Dickman et al. [37].

One of the models (TTP) we study here has some similarities with a SOC model introduced by Manna [38] (namely the rule for evolution of sites with double occupation) and also with a forest-fire model (FFM) [39]. Manna's model, like the one of BTW, has a thresh- 
old above which sand topples. This model also presents avalanches of all sizes, which obey a power-law size distribution.

Another model to be studied is the parityconserving dimer model (PCD), which is known to belong to a different universality class - the parityconserving universality class (PC). The dynamic rules are such that there is parity conservation in the number of dimers.

Previous studies by Dickman et al. [34] using scaling analysis yielded new relations between critical exponents governing avalanches and the ones of critical phenomena in systems with many absorbing states. In this paper we use numerical simulations to study such relationships, and test scaling laws. Also in this case exponents will be obtained for avalanches and compared with ones for time-dependent evolution through scaling laws.

Hereafter, we will consider only two models (TTP and PCD) to illustrate the relationship between systems with IMAS and SOC. Other models exist in the literature with the same characteristics [30] [40], however we chose these two because they are more familiar to the author.

\section{The models}

As mentioned before, we will present studies of two onedimensional models with multiple absorbing configurations, and obeying different kinds of conservation laws (with parity conservation (PC) and without (DP)). The former consists in a model of dimers whose dynamic rules will be defined below (PCD) [41]. The latter is the so-called threshold transfer process (TTP) [42], in which each site of the lattice can be either vacant, or singly or doubly occupied ( $\sigma_{i}=0,1,2$, respectively). At each time step, a site $i$ is chosen at random. In the absence of active (doubly occupied) sites the dynamics is indeed trivial: if $\sigma_{i}(t)=0(1)$, then $\sigma_{i}(t+1)=1(0)$ with probability $p(1-p)$. The system relaxes exponentially to a steady state where a fraction $p$ of sites have $\sigma_{i}=1$ and the others are vacant. If $\sigma_{i}(t)=2$, then $\sigma_{i}(t+1)=0, \sigma_{i-1}(t+1)=\sigma_{i-1}(t)+1, \sigma_{i+1}(t+1)=$ $\sigma_{i+1}(t)+1$ if $\sigma_{i+1}(t)$ and $\sigma_{i-1}(t)$ are both $<2$ and $\sigma_{i}(t+1)=1$ if only one of the nearest neighbours of site $i(j=i-1$ or $i+1)$ has $\sigma_{j}(t)<2$, in which case $\sigma_{j}(t+1)=\sigma_{j}(t)+1$. As can be easily seen, the number of active sites either decreases or remains the same in all processes other than $(1,2,1) \rightarrow(2,0,2)$; the frequency of this process depends on the concentration of ' 1 '-s, which is controlled by the parameter $p$. Any configuration consisting of only ' 0 '-s or ' 1 '-s is absorbing in what concerns the active sites. Thus in a chain of length $\mathrm{N}$, there are $2^{N}$ absorbing configurations. The absorbing states in this model are fluctuating - in this subsector of phase space, ergodicity is not broken. Thus we identify the density of doubly-occupied sites, $\rho_{2}$, as the order parameter of the threshold transfer process. Under the correspondence: $2 \rightarrow$ burning tree, $1 \rightarrow$ live tree, and $0 \rightarrow$ ashes, the process $(1,2,1) \rightarrow(2,0,2)$ describes a burning tree setting its neighbours on fire. But the TTP permits doubly occupied sites to arise only via transfer; there is no "lightning" process, as in the FFM. Despite certain common aspects, our model is therefore very different from the FFM.

The other model to be considered here was introduced by Marques et al. [41] and is the first to combine parity conservation (PC) with infinitely many absorbing states. The original idea of this model was to clarify whether, as in the TTP, PCP and other models without $\mathrm{PC}$ conservation, dynamic critical exponents will also depend on the initial state. This model resembles the PCP introduced by Jensen [30] but with the number of dimers conserved modulo 2. The dynamic evolution of this model involves creation and annihilation processes, which are attempted with probabilities $p$ and $1-p$ respectively. If one chooses to annihilate, then a dimer (represented by $\bullet=\bullet$ ) is selected at random and one looks for an adjacent dimer. If the pair of dimers is surrounded by empty sites (o), then the two dimers are annihilated and the respective sites become empty:

$-0-\bullet=\bullet=\bullet-0-\longrightarrow-0-0-0-0-0-$

otherwise, annihilation is effected by simply vacating the site that is shared by the two dimers, leaving the other sites unchanged:

$-\circ-\bullet=\bullet=\bullet=\bullet-\longrightarrow-\circ-\bullet-\circ-\bullet=\bullet-$.

In this way, the number of dimers is conserved modulo 2. In case of a creation attempt, if the nearestand next-nearest-neighbor sites of a selected dimer are, respectively, vacant and occupied, a pair of dimers is produced by simply occupying that vacant site:

Another possibility requires three vacant sites adjacent to a dimer, in which case a pair of dimers is created (with probability $\alpha$ ) by filling the nearest- and nextnearest neighbour sites and leaving the third vacant site unchanged:

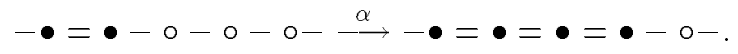

In the cases with $\alpha<1$, growth is less effective if there are vacant sites (rather than isolated particles) in the environment of the dimers, whereas in the case $\alpha=1$ growth is not affected by the concentration of isolated particles in the environment. In the present 
work, we consider $\alpha=1 / 2$. We should also point out that this model presents another conservation law, in addition to parity conservation. Define sublattices $A$ and $B$, and let $N_{A B}\left(N_{B A}\right)$ be the total number of dimers with their left particle on the $A(B)$ sub-lattice. Then $N=N_{A B}-N_{B A}$ is conserved, since dimers are created and annihilated in pairs on adjacent pairs of sites. This implies that configurations with different values of $N$ are not connected by the dynamics used.

With these rules, diffusion is only present indirectly. Also, the annihilation of isolated dimers is possible only through successive processes of creation and annihilation, which slows down the dynamical processes leading to absorbing configurations. One can of course enlarge the parameter space to include diffusion or longer range processes and consider a dynamics that mixes sectors in the phase space with different values of $N$. In the absence of such processes, creation of dimers is inhibited in configurations where a pair of vacant sites is surrounded by occupied sites. Initial configurations were chosen such that this situation does not occur in the dynamical process; this means a restriction to the $N=0$ sector.

\section{Summary of previous results}

In this section we present briefly results from [41] and [42]. In these two models with infinitely many absorbing configurations, it was shown that the static exponents belong to the corresponding universality class, that is, PC for PCD, and DP for TTP. In what concerns the spreading exponents, it was observed in both models that they depend on the initial configuration. To account for this a new hyperscaling relation was proposed [42]. This consists in a generalisation of the one proposed by Grassberger and de la Torre [43], and assumes that the ultimate survival probability behaves like $P_{\infty} \sim \Delta^{\beta^{\prime}}$.

\section{A. Mean-field theory}

The mean-field theory for the TTP model can be easily obtained. Defining by $\rho_{i}$ the densities for the three kinds of site occupation, we arrive at

$$
\frac{d}{d t} \rho_{i}=\sum_{k} R^{(k)} \Theta_{i}^{(k)} P^{(k)}
$$

where $R^{(k)}, \Theta_{i}^{(k)}$ and $P^{(k)}$ are respectively the rate for the process $k$, the change in the number of particles of type $i$, and the probability of the configuration required for process $k$.

\begin{tabular}{ccrrrc}
\hline Process & $R^{(k)}$ & $\Theta_{0}$ & $\Theta_{1}$ & $\Theta_{2}$ & $P^{(k)}$ \\
\hline $1 \rightarrow 0$ & $1-p$ & 1 & -1 & 0 & $\rho_{1}$ \\
$0 \rightarrow 1$ & $p$ & -1 & 1 & 0 & $\rho_{0}$ \\
$121 \rightarrow 202$ & 1 & 1 & -2 & 1 & $\rho_{2} \rho_{1}^{2}$ \\
$020 \rightarrow 101$ & 1 & -1 & 2 & -1 & $\rho_{2} \rho_{0}^{2}$ \\
$221 \rightarrow 212$ & 1 & 0 & 1 & -1 & $\rho_{1} \rho_{2}^{2}$ \\
$021 \rightarrow 102$ & 1 & 0 & 0 & 0 & $\rho_{0} \rho_{1} \rho_{2}$ \\
$220 \rightarrow 211$ & 1 & -1 & 2 & -1 & $\rho_{0} \rho_{2}^{2}$ \\
$222 \rightarrow 222$ & 1 & 0 & 0 & 0 & $\rho_{2}^{3}$ \\
\hline
\end{tabular}

TABLE I. Rules for the TTP model, and the respective rate processes[44].

Using the results of Table I, the dynamic equations for the densities appear in the form:

$$
\begin{aligned}
& \frac{d \rho_{0}}{d t}=-p \rho_{0}+(1-p) \rho_{1}-\rho_{0}^{2} \rho_{2}+\rho_{1}^{2} \rho_{2}-2 \rho_{0} \rho_{2}^{2} \\
& \frac{d \rho_{1}}{d t}=p \rho_{0}-(1-p) \rho_{1}+2 \rho_{0}^{2} \rho_{2}-2 \rho_{1}^{2} \rho_{2}+4 \rho_{0} \rho_{2}^{2}
\end{aligned}
$$

where $\rho_{2}=1-\rho_{0}-\rho_{1}$. This analysis leads to a $p_{c}=1 / 2$ and $\beta=1$. It allows us to get information about the transition, however it is quantitatively wrong. In Fig. 1 we show the phase diagram in the mean-field approximation and from numerical simulations.
Based on these equations it is possible to write down a field-theory (or set of Langevin equations), for the density of particles $\left(\rho_{1}\right)$ and of active sites $\left(\rho_{2}\right)$ based on symmetry principles. This type of analysis was introduced by Muñoz et al. [31, 45] for models with IMAS,

$$
\begin{aligned}
& \frac{\partial \rho_{1}(x, t)}{\partial t}=r_{1} \rho_{1}+c_{1} \nabla_{x}^{2} \rho_{1}-u_{1} \rho_{2}^{2}-w_{1} \rho_{1} \rho_{2}+\rho_{1}^{1 / 2} \eta_{1} \\
& \frac{\partial \rho_{2}(x, t)}{\partial t}=r_{2} \rho_{2}+c_{2} \nabla_{x}^{2} \rho_{2}-u_{2} \rho_{2}^{2}-w_{2} \rho_{1} \rho_{2}+\rho_{2}^{1 / 2} \eta_{2}
\end{aligned}
$$


where the coeficients $c_{i}, r_{i} u_{i}$ and $w_{i}$ are all constants depending on the microscopic parameter of the system, $p$, and $\eta_{i}$ are Gaussian white noise variables, obeying, $\left\langle\eta_{i}(x, t) \eta_{j}(x, t)\right\rangle=D_{i, j} \rho_{2}(x, t) \delta\left(x-x^{\prime}\right) \delta\left(t-t^{\prime}\right)$. This analysis has yet to reproduce the nonuniversal spreading exponents observed in models with INAS. This set of equations can be reduced to a single equation (for $\rho_{2}$ ) with an exponential non-Markovian term,

$$
\begin{aligned}
\frac{\partial \rho_{2}}{\partial t}= & D \quad \nabla^{2} \rho_{2}-r_{1} \rho_{2}-u_{2} \rho_{2}^{2}+\eta_{2} \\
& -\quad w_{2} r_{1} \rho_{2}(x, t) \int_{0}^{t} d t^{\prime} \rho_{2}\left(x, t^{\prime}\right) e^{-w_{1} \int_{t^{\prime}}^{t} d s \rho_{2}(x, s)}
\end{aligned}
$$

with a long-memory effect [31]. The last term on the right side is irrelevant to the stationary properties of the active phase, because it goes exponentially to zero if the density of active sites is non-null. This equation is different from the equation that describes Reggion field theory [48, 49, 50]. A numerical analysis was later made by Lopéz et al. [51]. Numerical integration shows the nonuniversal dynamic critical exponents observed previously for the PCP and other models with INAS.
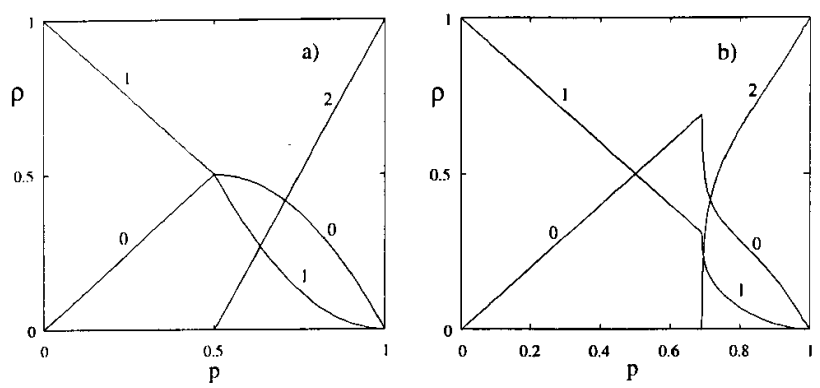

Figure 1. Density versus $p$ for the TTP model. Left: meanfield theory; right: Monte Carlo simulations. The labels on top of curves $(0,1,2)$ mean the densities of vacant, particles and pairs respectively.

\section{B. Steady-state simulations}

To perform these simulations we started with a fully occupied lattice in the case of PCD, and with equal fractions of the three states in the case of TTP. The concentrations are averaged over a long period of time, once the steady state has been reached. As noted above, the order parameter for the TTP is the density of 2's $\left(\rho_{2}\right)$; it obeys

$$
\rho_{2}=\left|p-p_{e}^{T T P}\right|^{\beta}
$$

The critical value is $p_{c}^{T T P}=0.6894 \pm 0.0003$. The exponent $\beta$ was measured previously for this model [42], and is in very good agreement with the DP universality class $(d=1+1)$. In simulations we found $\beta=0.279 \pm 0.005$ [42].
The order parameter for the PCD is the concentration of dimers; it too vanishes algebraically as $p$ approaches the critical probability $p_{c}$ :

$$
\rho \sim\left|p_{c}^{P C D}-p\right|^{\beta}
$$

where $\beta$ is the order parameter exponent $\left(p_{c}^{P C D}=\right.$ $0.2995 \pm 0.0005)$. Simulations yield $\eta=0.285$, in agreement with the value for the PC universality class in $1+1-d$.

As usual, the steady-state concentration is obtained for several values of $p$ near $p_{c}$, and different values of system size, up to a maximum value of $L=2000$. The maximum duration was chosen according the value of $p$; studies near $p_{c}$ require long durations, because of slowing-down effects. We used $10^{4}<t<10^{6}$. The simulations were carried out for about 1000 independent trials which had not yet entered the absorbing state. From a log-log plot of density against $p-p_{c}$, the slope of the data gives the estimate $\beta=0.98(5)$, a value which agrees with the results of [52], and is slightly above the values obtained by other authors for $(1+1)-d$ models in the PC universality class. Determining the critical point by steady-state simulations requires an accurate measurement of the order parameter near criticality, which becomes rather difficult due to the critical slowing-down. We have complemented this study with a finite-size scaling analysis based on the ansatz that the order parameter depends on system size $L$ through the ratio of $L$ and the correlation length $\xi_{\perp} \sim \Delta^{-\nu_{\perp}},\left(\Delta=\left|p-p_{c}\right|\right)$ :

$$
\rho(p, L) \sim L^{-\beta / \nu_{\perp}} f\left(\Delta L^{1 / \nu_{\perp}}\right)
$$

(with $f(x) \propto x^{\beta}$ for $x \rightarrow \infty$, so that (1) is recovered when $L \rightarrow \infty)$. At the critical point we have,

$$
\rho\left(p_{\iota}, L\right) \propto L^{-\beta / \nu_{\perp}}
$$

and from a log-log plot of $\rho\left(p_{c}, L\right)$ as a function of $\mathrm{L}$; a linear dependence is obtained, with slope $\beta / \nu_{\perp}=$ $0.54(4)$, in good agreement with the values 0.48 and 0.50 , as obtained in [53] and [54].

The ultimate survival probability exponent $\beta^{\prime}$ was determined from similar studies, using $p$ values slightly away from the critical point. The numerical procedure will be explained in the next section.

\section{Time-dependent simulations}

Time-dependent simulations [43] have become an effective tool to explore dynamical critical exponents of systems at nonequilibrium phase transitions.

Initial Configuration. Simulations are run for a large number of trials; for each of them we construct an initial configuration with a single active seed (a dimer in 
the case of PCD, or a doubly-occupied site in the case of TTP), embedded in a sea of inactive sites (vacant or singly-occupied sites), i.e, $(\cdots-\circ-\bullet-\circ-\bullet=$ - $-\circ-\circ-\circ-\bullet-\cdots)$ located on the center of the system (sites $L / 2$ and $L / 2+1$ for a lattice of $L$ sites). We place a given concentration $\phi_{1}$ of particles on the other sites, with the condition (in the case of the PCD) that there are no nearest-neighbor pairs (i.e., no dimers other than the central seed). For the PCD model the maximum initial density is $1 / 2$; for TTP it is 1 .

Temporal evolution. We set a maximum time for the evolution $\left(t_{M A X}\right)$ chosen in such a way that active sites cannot reach the borders of the system. In this way there are no finite size effects. Since the number of active particles during the time evolution is small, we save CPU time by choosing from a list of the active sites and then applying the the process (creation or annihilation). This procedure entails a variable time increment, $\Delta t=1 / N_{\text {act }}$, for each event, where $N_{\text {act }}$ is the current number of active sites. The quantities usually investigated are the mean number of active sites $N(t)$ (dimers in case of PCD and '2'-s in case of TTP model), averaged over all trials, the survival probability $P(t)$, and the mean spread or size, $R(t)$, of the surviving trials. Near the critical point, and for asymptotically long times these quantities follow power laws of the form

$$
\begin{aligned}
N(t, \Delta) & =\int d x \rho(x, t, \Delta)=t^{\eta} \Omega\left(t \Delta^{\nu_{\|}}\right) \\
P(t, \Delta) & =t^{-\delta} \Psi\left(t \Delta^{\nu} \|\right) \\
R^{2}(t, \Delta) & =\frac{1}{N(t, \Delta)} \int d x r^{2} \rho(x, t, \Delta)=t^{z} \Phi\left(t \Delta^{\nu_{\|}}\right)
\end{aligned}
$$

where $x$ is the distance from the seed and the normalisation quantity $N(t, \Delta)$ is the mass of the cluster at time $t$. The latter expressions define the exponents $\eta$, $\delta$ and $z$ respectively.
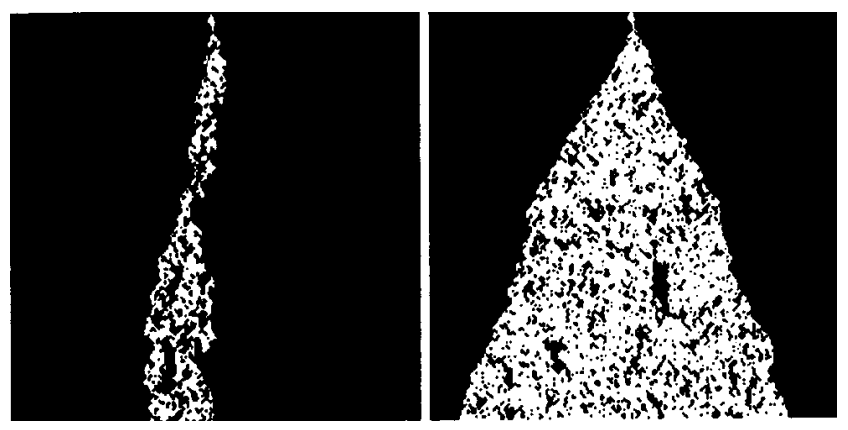

Figure 2. Ilustration of space-time evolution: below and above $p_{c}$.

It was observed by Mendes et al. [42] that $P_{\infty}$ (ultimate survival probability) as a function of $p-p_{c}$ for several initial densities is no longer proportional to the density, but is characterised by a new exponent $\beta^{\prime}$, $P_{\infty} \propto \Delta^{\beta^{i}}\left(\Delta=\left|p-p_{c}\right|\right)$. From this it follows that the density is

$$
\rho(x, t)=P_{\infty}(\rho) \Delta^{\beta} .
$$

Using this assumption one can obtain a hyperscaling relation [42], that generalises the one of Grassberger and de la Torre [43],

$$
\left(1+\frac{\beta}{\beta^{\prime}}\right) \delta^{\prime}+\eta^{\prime}=\frac{d z^{\prime}}{2}
$$

where the primes denote exponents dependent upon the initial particle density, $\phi$. This relation has been verified for a vast variety of models in different universality classes [41] [42] [31] [51] [46].

We have verified Eq. (11) in simulations of the parity-conserving dimer reaction and the threshold transfer process. Using time-dependent simulations (for $t \leq 5000$, and a number of trials of order $10^{7}$ ), we determined the critical point of the TTP as $p_{c}=0.6894(3)$. We note in passing that some controversy exists regarding a possible shift of $p_{c}$ as the initial density is varied, in models with INAS. A recent work by Dickman [55], using a new technique applied to the pair contact process (PCP), claims that $p_{c}$ is not affected by the initial concentration, contrary to what was seen by Ódor et al. [47]. The exponents $\delta^{\prime}, \eta^{\prime}$ and $z^{\prime}$ may be determined from simulations at $p_{c}$, using an initial configuration very close to the absorbing state. We studied various initial densities, including $\phi_{i}=0.6894$, the natural value. The ultimate survival probability exponent $\beta^{\prime}$ was determined from similar studies, using $p$ values slightly above critical. The simulations begin with one doubly-occupied site at the origin; the remaining sites are taken as occupied or vacant, independently, with probabilities $\phi_{i}$ and $1-\phi_{i}$, respectively. The dynamics is restricted to the active region, defined as follows. Let $\Omega_{i}$ be the set of all sites which are doubly-occupied or have a doubly-occupied neighbour, after the $i^{\text {th }}$ step of the trial. ( $\Omega_{0}$ comprises the origin and its neighbours.) The site to be updated at step $i+1$ is selected at random from $\cup_{j=0}^{i} \Omega_{j}$. Thus the evolution proceeds on an expanding set within the "light-cone" emanating from the origin. Distant sites are not updated until the active region reaches their neighborhood.

In these simulations, starting from a pair of dimers at the center of the lattice for the PCD and a 2 in the case of TTP, we measure the survival probability $P(t)$, the number of active particles (dimers or 2's) $N(t)$ (averaging over all runs), the average spread of the cluster $R^{2}(t)$, and the probability $\mathcal{P}(s)$ to have a cluster of size (mass) $s$, all at criticality. 
Spreading exponents are usually obtained from a local-slope analysis. For instance, from a plot of $\ln [P(m t) / P(t)] / \ln m$ vs. $t^{-1}$, we can get the exponent $\delta^{\prime}$. However a more recent analysis [55], shows that it is better to plot against $t^{-\delta_{D P}}$. This because in previous studies it was observed that the leading term in,

$$
\delta(t) \sim \delta\left[1+a t^{-\theta}+\cdots\right]
$$

was controled by $\theta=1$, however from the observations of Ódor el al. [47] the particle density evolves in time with a power-law decay controlled by an exponent equal to $\delta_{D P}$, thus suggesting a different leading correction term in $\delta(t)$.

We have carried out simulations at the critical probability $p_{c}$. In a plot of the local slopes versus $1 / t$, the critical exponents are given by the intercept of the curves for $p_{e}$ with the vertical axis, whereas curves for $p>p_{c}\left(p<p_{c}\right)$ veer downward (upward). From this analysis, we too do not see any shift in the critical value, $p_{c}$. Our results are listed in Table II.

One goal of this study is to obtain numerical confirmation of the exponents describing cluster-size distributions at criticality. The typical cluster size $s$ of finite clusters scales as volume times density, i.e.,

$$
s \sim \xi_{\perp}^{d} \xi_{\|} \rho(\Delta) \sim|\Delta|^{-1 / \sigma^{\prime}},
$$

with

$$
1 / \sigma^{\prime}=d \nu_{\perp}+\nu_{\|}-\beta^{\prime},
$$

where $\xi_{\perp}$ and $\xi_{\|}$are the correlation lengths in the space and time directions, respectively. From the lifetime survival distribution, Eq. (8), it is then straightforward to obtain the probability $\mathcal{P}(s)$ to have a cluster of size $s$, for clusters starting from a single seed. Using the fact that the lifetime is set by the parallel correlation length, $t \sim \xi_{\|} \sim|\Delta|^{-\nu} \|$, we see that the typical cluster size and lifetime are connected by

$$
s \sim t^{1 / \nu_{\|} \sigma^{\prime}}
$$

Hence we obtain $P(s) \sim P\left(t \sim s^{\nu} \|^{\sigma^{\prime}}\right) \sim s^{-\beta^{\prime} \sigma^{\prime}}$. Thus, we eventually obtain the probability $\mathcal{P}(s)$ to have a cluster of exactly size $s, \mathcal{P}(s)=-d P(s) / d s$, with the result

$$
\mathcal{P}(s) \sim s^{-\tau_{s}},
$$

where

$$
\tau_{s}=1+\frac{\beta^{\prime}}{d \nu_{\perp}+\nu_{\|}-\beta^{\prime}}=\frac{1+\eta^{\prime}+2 \delta^{\prime}}{1+\eta^{\prime}+\delta^{\prime}} .
$$

Proceeding in the same way, we get $\mathcal{P}(t)=$ $-d P(t) / d t \propto t^{-\left(1+\delta^{\prime}\right)}$, and thence,

$$
\tau_{t}=1+\delta^{\prime}
$$

In simulations we measure the probability of having a cluster of size $s$ in the critical state.

In Marques et al. [41] a slight shift in $p_{c}$ was observed in the time-dependent simulations for the PCD model, however a more precise analysis seems to suggest that this dependence may not exist. In a recent paper [55] a claim based on a more precise method (reweighting method [56]) states that in the case of PCP the critical parameter is not shifted when considering different initial configurations as was observed previously [47].

In these simulations we started the system in a way that one sublattice of alternating sites is vacant except for one central site, and the sites of the other sublattice are occupied with probability $\phi$; the two nearest neighbours of the central site are also occupied, thus constituting a perturbation of exactly two dimers. The size of the lattice was chosen such that activity never hits the boundaries. For different $\phi$ values, a number of independent runs, typically $10^{7}$, were performed, up to 4000 time steps each, for various values of $p$.

Results for the $\phi$-dependence of $\delta^{\prime}$ and $\eta^{\prime}$ are given in Table II. Indeed, the exponent $z^{\prime}$ does not present a significant dependence on the parameter $\phi$, given the numerical errors. The exponent governing the population growth in surviving critical trials, $\delta^{\prime}+\eta^{\prime}$, seems not to depend on the initial particle concentration; we do not see a systematic change with $\phi$. For the PCP, a recent study [55] suggests that this quantity, and the exponent $z$, have a systematic dependence upon $\phi$. The clarification of such results is a topic for further research, namely through the use of the reweighting method [56].

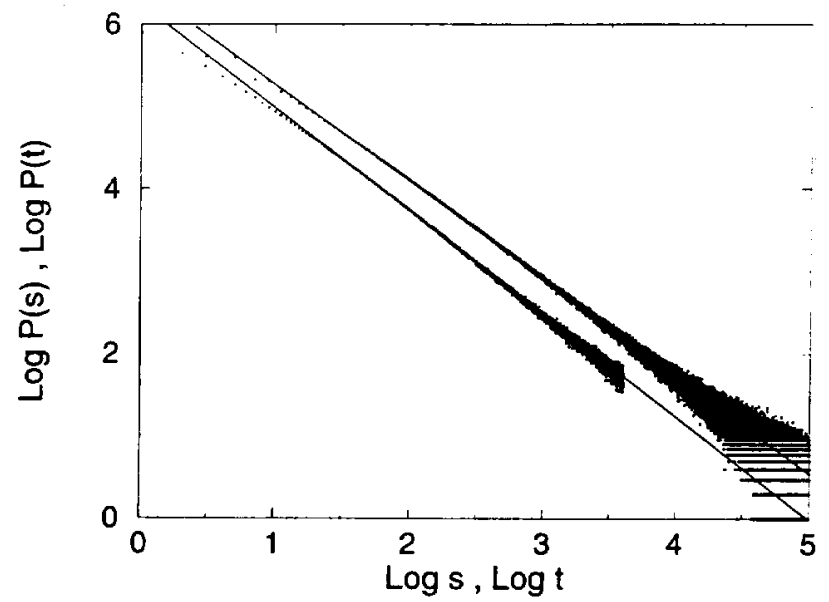

Figure 3. Log-log plot of the distributions $P(s)$ and $P(t)$ for the PCD model with an initial concentration of particles equal to 0.3 . The lower data correspond to the time distribution, which was reduced in order to not overlap with the upper one. The slope was measured considering all the data. 


\section{Numerical results for avalanche spreading}

When the system is tuned to its critical value, it develops avalanches obeying a power-law distribution. We will characterise the avalanche activity through two quantities, its spatial and temporal duration distributions:

$$
\begin{aligned}
& \mathcal{P}(s) \sim s^{-\tau_{s}} \\
& \mathcal{P}(t) \sim t^{-\tau_{t}}
\end{aligned}
$$

We have measured these avalanche distributions in both models. The simulation results are given in Table II. Fig. 3 shows (for a particular case, $\phi=0.3$, in the PCD model) a log-log plot of the distributions of space and time avalanche duration. The exponents obtained from the slopes of data points in this particular case are, $\tau_{s}=1.193 \pm 0.010$ and $\tau_{t}=1.256 \pm 0.010$. The values are in very good agreement with the ones obtained through the scaling laws, and using the values of the non-universal spreading critical exponents (see Table II), we get $\tau_{s}=1.184$ and $\tau_{t}=1.239$.

In Fig. 4 we plot the values for $\tau_{s}$ and $\tau_{t}$ for different values of $\phi$ in the PCD model. The lines correspond to the exponents obtained from the scaling laws.

From the previous studies we got the $p_{c}$ value for each model. In order to study avalanches we prepare the system in the initial state with some density of particles and $p=p_{c}$, then through the evolution of a single perturbation (adding a "critical" particle at the middle site) we will measure the propagation of this damage through the system. It will affect a certain number of sites - this is the avalanche size - and also has a certain lifetime - this is the time duration of the avalanche. In order to have a good precision in the avalanche exponents it is necessary to use a large number of trials (we used $2 \times 10^{7}$ ).

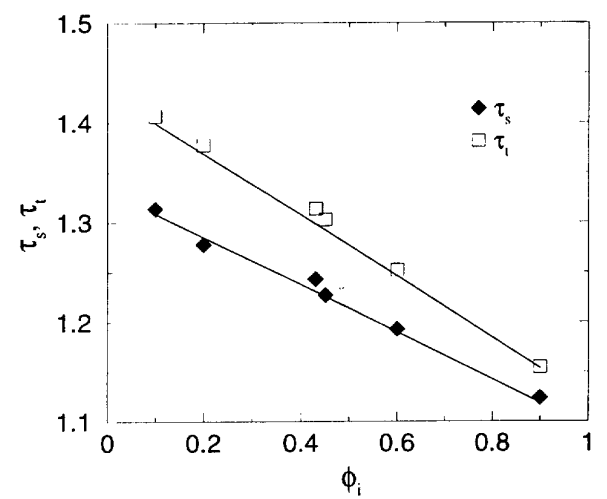

Figure 4. Avalanche exponents $\tau_{s}$ and $\tau_{t}$ versus initial density $\phi$ in the PCD model.

\section{Conclusion}

We reviewed some topics related to models with infinitely many absorbing states (namely, the PCD and TTP), and have presented some new results for avalanche spreading. The numerical values obtained by from Monte Carlo simulations are in good agreement with those obtained from scaling analysis. The models studied here belong to two different universality classes (DP and PC). The nonuniversal spreading exponents previously observed in models with INAS, when the initial configuration differs from the "natural" one, are also seen in the exponents that characterise both spatial and temporal distributions of avalanches.

This work was supported in part by PRAXIS XXI (Project /2/2.1/Fis/299/94) and NATO (Grant CRG970332). I would like to thank R. Dickman, S.N. Dorogovtsev, M. Henkel, H. Herrmann, E.J.S. Lage, M.C. Marques, G. Ódor and M.A. Santos for many usefull discussions, and from whom I have learned a lot. I would like to thank M.A Santos and M.C. Marques for

\begin{tabular}{|c|c|c|c|c|}
\hline Exponents & $\delta^{\prime}$ & $\eta^{\prime}$ & $\tau_{s}$ & $\tau_{t}$ \\
\hline$\overline{\mathrm{DP}}$ & $0.1597(3)$ & $0.312(2)$ & 1.108 & 1.159 \\
\hline $\mathrm{PC}$ & $0.285(2)$ & $0.000(1)$ & 1.222 & 1.285 \\
\hline \multicolumn{2}{|c|}{$\phi_{\text {initial }}$} & Conserving & \multicolumn{2}{|c|}{ Dimer $(P C D)$} \\
\hline 0.05 & $0.400(6)$ & $-0.106(5)$ & $1.313(10)$ & $1.407(10)$ \\
\hline 0.10 & $0.366(6)$ & $-0.069(5)$ & $1.278(10)$ & $1.385(10)$ \\
\hline 0.215 & $0.291(6)$ & $0.005(5)$ & $1.243(10)$ & $1.318(10)$ \\
\hline 0.225 & $0.284(6)$ & $0.014(5)$ & $1.227(10)$ & $1.303(10)$ \\
\hline 0.30 & $0.239(6)$ & $0.062(5)$ & $1.193(10)$ & $1.256(10)$ \\
\hline 0.45 & $0.156(6)$ & $0.154(5)$ & $1.124(10)$ & $1.169(10)$ \\
\hline \multicolumn{5}{|c|}{ Threshold Transfer Procces (TTP) } \\
\hline 0.75 & $0.136(1)$ & $0.347(4)$ & $1.093(10)$ & $1.14(2)$ \\
\hline 0.69 & $0.161(2)$ & $0.319(3)$ & $1.121(10)$ & $1.17(2)$ \\
\hline 0.50 & $0.227(2)$ & $0.246(2)$ & $1.157(10)$ & $1.20(2)$ \\
\hline 0.10 & $0.371(4)$ & $0.097(1)$ & $1.254(10)$ & $1.37(2)$ \\
\hline
\end{tabular}
a critical reading of the manuscript.

TABLE II. $\phi$-dependence of the critical exponents $\delta^{\prime}, \eta^{\prime}$ for spreading, and of $\tau_{s}$ and $\tau_{t}$, for the extent and duration of avalanches. 


\section{References}

[1] Percolation Structures and Processes, edited by G. Deutscher, R. Zallen, and J. Adler, Ann. Israel Soc.5 (Hilger,Bristol,1983).

[2] T.M. Liggett, Interacting Particle Systems, (SpringerVerlag, New York, 1985).

[3] J. Marro and R. Dickman, Nonequilibrium phase transitions in lattice models (Cambridge University Press, Cambridge, 1999).

[4] P. Grassberger, J. Phys. A 22, 3673 (1989).

[5] R. Dickman, Phys. Rev. B 40, 7005 (1989).

[6] R. Dickman, Phys. Rev. A 42, 6985 (1990).

[7] E. Domany and W. Kinzel, Phys. Rev. Lett. 53, 31 (1984).

[8] D. Stauffer and A. Aharony, Introduction to Percolation Theory (Taylor \& Francis, London, 1992).

[9] J. L. Cardy and R. L. Sugar, J. Phys. A 13, L423 (1980).

[10] I. Jensen, H. C. Fogedby, and R. Dickman, Phys. Rev. A 41, 3411 (1990).

[11] G. Grinstein, Z. W. Lai, and D. A. Browne, Phys. Rev. A 40, 4820 (1989).

[12] Braodbent and Hammersley, Proc. Camb. Philos. Soc. 53, $629(1957)$

[13] J. Blease, J. Phys. C 10, 923 (1977)

[14] J. W. Essam and K. De'Bell, J. Phys. A 14, L459 (1981)

[15] W. Kinzel, Z. Physik B 58, 229 (1985).

[16] R. Bidaux, N. Boccara, H. Chaté, Phys. Rev. A 39, 3094 (1989).

[17] D. ben-Avraham, J. Köhler, J. Stat. Phys. 65, 839 (1991).

[18] H. Takayasu, A. Yu. Tretyakov, Phys. Rev. Lett. 68, 3060 (1992).

[19] T. E. Harris, Ann. Prob. 2, 969 (1974).

[20] R. Dickman, J. Stat. Phys.55, 997 (1989).

[21] I. Jensen, Phys. Rev. A 43, 3187 (1991).

[22] R.M. Ziff, E. Gulari, Y. Barshad, Phys. Rev. Lett. 56, $2553(1986)$

[23] M. Alon, M. R. Evans, H. Hinrichsen and D. Mukamel, Phys. Rev. Lett. 76, 2746 (1996).

[24] S. Maslov, M. Paczuski and P. Bak, Europhys. Lett. 27, 97 (1994).

[25] H. K. Jenssen, Z. Phys. B 42, 151 (1981)

[26] P. Grassberger, Z. Phys. B 47, 365 (1982).

[27] G. Grinstein, C. Jayaprakash and Y. Hu, Phys. Rev. Lett. 55, 2527 (1985).

[28] P. Grassberger, F. Krause, T. von der Twer, J. Phys. A 17, L105 (1984).
[29] P. Grassberger, J. Phys. A 22, L1103 (1984).

[30] I. Jensen, Phys. Rev. Lett. 70, 1465 (1993), I. Jensen, Phys. Rev. E 427, R1 (1993).

[31] M. A. Muñoz, G. Grinstein, R. Dickman and R. Livi, Phys. Rev. Lett. 76, 451 (1996)

[32] A. Lipowski, cond-mat/9910029; A. Lipowski and M. Loparta, Phys. Rev. E 60, 1516 (1999).

[33] G. Parisi and N. Sourlas, Phys. Rev. Lett. 43, 744 (1979).

[34] R. Dickman, A. Vespignani, S. Zapperi, Phys. Rev. E 57, 5095 (1998)

[35] A. Vespignani, R. Dickman, M.A. Muñoz and S. Zapperi, Phys. Rev. Lett 81, 5676 (1998)

[36] P. Bak, C. Tang and Weisenfeld, Phys. Rev. Lett. 59, 381 (1987).

[37] R. Dickman, M. Muñoz and A. Vespignani, S. Zapperi. This volume.

[38] S.S. Manna, J. Phys. A 24, L363 (1991).

[39] B. Drossel, Phys. Rev. Lett. 76, 936 (1996).

[40] I. Jensen, R. Dickman, Phys. Rev. E 48, 1710(1993).

[41] M.C. Marques and J.F.F. Mendes, Eur. Phys. J. B 12, $123(1999)$.

[42] J.F.F. Mendes, R. Dickman, M. Henkel, M.C. Marques, J. Phys. A 27, 3019 (1994)

[43] P. Grassberger and de la Torre, Ann. Phys. 122, 373 (1979).

[44] J.F.F. Mendes, Phd thesis (1994).

[45] M. A. Muñoz, G. Grinstein and R. Dickman, J. Stat. Phys. 91, 541 (1998)

[46] R. Dickman, Phys. Rev. E 53, 2223 (1996).

[47] G. Ódor, J.F.F. Mendes, M.A. Santos, M.C. Marques, Phys. Rev. E 58, 7020 (1998)

[48] V. N. Gribov, Sov. Phys. JETP 26, 414 (1968)

[49] P. Grassberger and K. Sundermeyer, Phys. Lett. B 77, 220 (1978).

[50] R. C. Brower, M. A. Furman and M. Moshe, Phys. Lett. B 76, 213 (1978).

[51] C. Lopez and M.A. Muñoz, Phys. Rev. E 56, 4864 (1997).

[52] N. Inui and A. Yu Tretyakov, Phys. Rev. Lett. 80, 5148 (1998).

[53] Mann Ho Kim, Hyunggyu Park, Phys. Rev. Lett. 73, 2579 (1994); Heungwon Park, Mann Ho Kim, Hyunggyu Park, Phys. Rev. E 52, 5664 (1995).

[54] I. Jensen, Phys. Rev. E 50, 3623 (1994).

[55] R. Dickman, cond.mat/9909347.

[56] R. Dickman, Phys. Rev. E 60, R2441 (1999). 